

\title{
Interferometric Characterization of Subwavelength Lamellar Gratings
}

Philippe Lalanne, Pierre Pichon, Pierre Chavel, Edmond Cambril, Huguette Launois

\section{- To cite this version:}

Philippe Lalanne, Pierre Pichon, Pierre Chavel, Edmond Cambril, Huguette Launois. Interferometric Characterization of Subwavelength Lamellar Gratings. Applied optics, 1999, 38 (23), pp.4980-4984. 10.1364/AO.38.004980 . hal-00877945

\section{HAL Id: hal-00877945 \\ https://hal-iogs.archives-ouvertes.fr/hal-00877945}

Submitted on 29 Oct 2013

HAL is a multi-disciplinary open access archive for the deposit and dissemination of scientific research documents, whether they are published or not. The documents may come from teaching and research institutions in France or abroad, or from public or private research centers.
L'archive ouverte pluridisciplinaire HAL, est destinée au dépôt et à la diffusion de documents scientifiques de niveau recherche, publiés ou non, émanant des établissements d'enseignement et de recherche français ou étrangers, des laboratoires publics ou privés. 


\title{
Interferometric characterization of subwavelength lamellar gratings
}

\author{
Philippe Lalanne, P. Pichon, P. Chavel, E. Cambril, and H. Launois
}

\begin{abstract}
We propose a new, to our knowledge, method for determining the two main critical parameters of periodic one-dimensional lamellar structures, namely, linewidths and etched depths. The method is simple and requires only two measurements for the phase of the zero-transmitted order under two orthogonal polarizations. It is inspired by the analogy between subwavelength gratings and anisotropic homogeneous thin films. The method is tested with experimental data obtained with a Mach-Zehnder interferometer. Etched depths and linewidths derived from the interferograms and electromagnetic theory are compared with scanning-electron-microscope observations. (C) 1999 Optical Society of America

OCIS codes: $\quad 050.1380,050.1970,050.1950,290.3200$.
\end{abstract}

\section{Introduction}

The steps leading to diffractive components involve a design phase that takes technological limitations into account, a fabrication phase, and a final phase of the component testing. With current nanofabrication facilities, linewidths and periods of the order of a few tens or hundreds of nanometers are manufacturable. For the determination of the grating profiles, for example, scanning electron microscopy is the primary reference method. This approach is obviously time consuming, destructive (in general, the wafer has to be cleaved and metallized), and limited to a small subset of the diffractive components or of the etched wafer. A well-known method for nondestructive testing based on the measurement and analysis of light scattered from a corrugated surface is optical scatterometry. In the case of periodic structures, light scattering takes the form of diffraction into orders. The information about the structure is routinely obtained from the distribution of intensities in the various diffracted orders. The inverse problem, which consists of reconstructing the grating geometry

Ph. Lalanne (philippe.lalanne@iota.u-psud.fr), P. Pichon, and P. Chavel are with the Laboratoire Charles Fabry de l'Institut d'Optique, Centre National de la Recherche Scientifique, B.P. 147, F-91403 Orsay cedex, France. E. Cambril and H. Launois are with the Laboratoire de Microstructures et de Microélectronique, Centre National de la Recherche Scientifique, 196 avenue Henri Ravera, B.P. 107, F-92225 Bagneux, France.

Received 27 January 1999; revised manuscript received 23 April 1999.

0003-6935/99/234980-05\$15.00/0

(C) 1999 Optical Society of America from the observed far-field data, is ill posed in the sense of Hadamard. ${ }^{1}$ In practice, a priori knowledge is introduced, and the grating profile is described by a finite number of parameters. Many independent measurements are performed by means of scanning over the angles of incidence and wavelength and by use of two orthogonal polarizations. One chooses the geometry that corresponds to the smallest deviation from the measured diffraction data. In diffractive optics this approach has been applied to a large variety of problems including, for example, metallic gratings, ${ }^{2}$ large-period gratings, ${ }^{3}$ and volume gratings. ${ }^{4}$ More recently, ellipsometric arrangements allowing for additional information from the phase of the diffracted orders were used by Cui and Azzam ${ }^{5}$ to characterize blazed metallic gratings and by Giovannini et al. ${ }^{6}$ to characterize holographic sinusoidal aluminum gratings by exploitation of plasmon resonances. In semiconductor metrology, ${ }^{7}$ intensity measurements and ellipsometry are now widely employed for in situ control and monitoring of growth and etching of periodic silicon surfaces (see, for example, Refs. 8 and 9). Future trends in that field are driven by the decrease of device dimensions with sub$0.1-\mu \mathrm{m}$ linewidths. ${ }^{10}$

Hereafter we focus on subwavelength onedimensional lamellar gratings with one groove per period. The grating period is known and fixed by the fabrication process, holographic recording, or electronbeam (e-beam) writing. It is smaller than the wavelength of the probing light and is typically of the order of a few hundreds of nanometers. Only the transmitted and the reflected zero orders propagate in the far field. The grating is assumed to be etched in a known 
material with a relative permittivity denoted by $\epsilon$. Thus only two parameters have to be estimated, namely, the linewidth and the etched depth. In this paper an interferometric technique for estimating these two parameters is proposed. It is described in Section 2. The technique is simple and requires only two measurements: the phases of the transmitted zero orders under two orthogonal polarizations. In Subsection 2.A the static limit, periods infinitely small compared with the wavelength of the probing light, is first considered. It is shown that, in this asymptotic case, knowledge of the phases of the zero-transmitted orders under two orthogonal polarizations is sufficient to determine unambiguously the two grating parameters. In Subsection 2.B practical situations for which the period is small compared with the wavelength but not infinitely small are considered. Experimental results obtained with a Mach-Zehnder interferometer are reported for several gratings etched in the same film in Section 3. The estimated linewidths and etched depths are compared with those obtained from scanning-electron-microscope (SEM) photographs. Section 4 provides a discussion and concludes the paper.

\section{Principle of the Method}

\section{A. Asymptotic Case}

In this section the proposed interferometric method is introduced through the analysis of an asymptotic case with grating periods infinitely small compared with the wavelength of the probing light. In this static limit it has been demonstrated ${ }^{11}$ that the grating is mathematically equivalent to a birefringent thin film. Thus, when a plane wave is normally incident onto the grating, the phase shift $\phi_{\mathrm{TE}}$ of the zero-order transmitted wave is approximately ${ }^{12}$

$$
\phi_{\mathrm{TE}}=(2 \pi / \lambda)\left(n_{\mathrm{TE}}-1\right) h
$$

for TE polarization (electric field parallel to the groove). Similarly, for TM polarization (magnetic field parallel to the groove), the phase shift $\phi_{\mathrm{TM}}$ is

$$
\phi_{\mathrm{TM}}=(2 \pi / \lambda)\left(n_{\mathrm{TM}}-1\right) h .
$$

In Eqs. (1) and (2) $h$ is the grating depth; $\lambda$ is the wavelength in the vacuum of the probing light; and $n_{\mathrm{TE}}$ and $n_{\mathrm{TM}}$ represent the static effective indices of the grating for TE and TM polarizations, respectively. For the sake of illustration, Fig. 1 shows the TE-TM phase-shift ratio as a function of the fill factor for a binary grating etched in a material whose relative permittivity is $\epsilon=5.29$. Of importance is the fact that this phase-shift ratio varies monotonously with the fill factor. This is a general result that can easily be seen to hold for any lamellar grating in the static limit. Thus, from the measurement of this ratio, the fill factor can be determined. Now, for a given fill factor, the phase-shift difference $\phi_{\mathrm{TE}}-\phi_{\mathrm{TM}}$ varies linearly with the grating depth. Thus, from the measurement of this difference, the grating depth can be determined. We conclude that the measure-

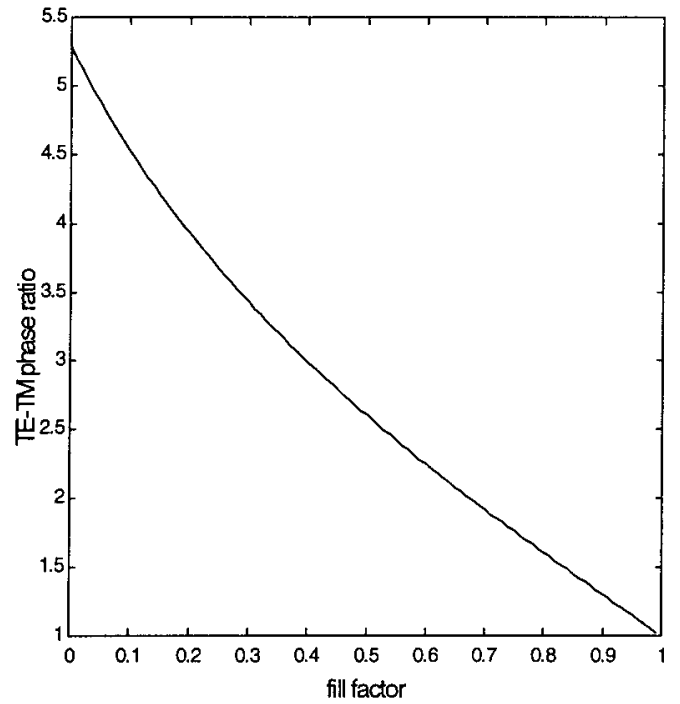

Fig. 1. TE-TM phase-shift ratio in the static limit as a function of the fill factor for a grating etched in a material with a relative permittivity $\epsilon=5.29$.

ment of the phase shifts for TE and TM polarizations under normal incidence is sufficient to unambiguously determine the fill factor and depth of dielectric lamellar gratings in the static limit. Under the $a$ priori assumptions of vertical walls and of known period and permittivity this analysis shows that the inverse problem is well posed: The solution exists, is unique, and depends continuously on the experimental data. In particular, the solution is stable with respect to small variations of the measured data.

\section{B. Real Cases}

In real situations the period is not infinitely small compared with the wavelength; the equivalence between gratings and homogeneous thin films is not rigorous, and the previous conclusions no longer hold strictly. However, from continuity arguments, it could reasonably be expected that the proposed method may be applied to gratings with periods of the order of a fraction of wavelengths for which Eqs. (1) and (2) are approximately valid. In these situations an analogy between subwavelength gratings and homogeneous thin films exists. Let us summarize the conditions under which this analogy is legitimate:

1. The grating period must be smaller than the cutoff value equal to the wavelength of the probing light in the substrate for normal incidence. This amounts to considering that all the diffracted orders (except the transmitted and reflected zero orders) are evanescent.

2. The grating period must also be smaller than the structural cutoff value, ${ }^{13}$ defined as the period value below which only one fundamental mode propagates in the grating region. If this condition is not fulfilled, the solution may be unstable with respect to small variations of the measured data ${ }^{14}$ and other 
measurements by scanning over the angle of incidence or the wavelength are required.

3. The grating depth must be larger than a quater wave. This amounts to considering that evanescent waves in the grating region have a negligible impact on the phase of the transmitted zero order. ${ }^{15}$ More specifically, since the birefringence $n_{\mathrm{TE}}-n_{\mathrm{TM}}$ of subwavelength gratings vanishes in the limit of infinitely small depths, the proposed method, which relies on the measurement of this birefringence, may fail.

Clearly, these are arguments based only on physical considerations. In general the existence, uniqueness, and stability of the solution are not guaranteed, but we believe that the proposed method remains qualitatively valid and useful for subwavelength gratings. The following illustrates this point with experimental data.

\section{Experiments}

To validate the proposed method, we fabricated five $200 \mu \mathrm{m} \times 200 \mu \mathrm{m}$ subwavelength lamellar gratings on the same substrate. The fabrication process involves $e$-beam lithography in a poly(methyl methacrylate) film, lift-off with an intermediate nickel layer, and reactive ion etching of a $\mathrm{TiO}_{2}$ film (refractive index 2.3) evaporated on a glass substrate (refractive index 1.52). The lift-off technique is used to enhance the selectivity and the fidelity of the pattern transfer during the etching. Nearly vertical walls are thus implemented. All the grating periods are equal to $448 \mathrm{~nm}$. The period is controlled accurately by the $e$-beam writing process. More details concerning the fabrication and the application of such gratings as polarizing beam splitters at $633 \mathrm{~nm}$ can be found in Ref. 16.

We varied the dose of the $e$-beam around a nominal value; the five gratings were fabricated for several dose deviations $(-15 \%, 0 \%, 20 \%, 40 \%$, and $60 \%)$. Because of the lift-off, large doses are expected to result in large linewidths. The gratings were incorporated into one of the arms of a Mach-Zehnder interferometer and the interference patterns were recorded on a CCD camera for two orthogonal polarizations at $633 \mathrm{~nm}$. Figure 2 shows two typical interferograms that were obtained for one of the gratings under TE and TM polarizations. From the interferograms the phase shifts are measured. ${ }^{17}$

The simplified approach in the static limit was first applied to quickly derive a rough estimation for $f$ and $h$. Then, because neither condition 1 nor 2 is satisfied for the specific case considered here, we performed an extensive exploration in the configuration space for refinements, using electromagnetic theory, and we computed the phase shifts $\Phi_{\mathrm{TE}}(f, h)$ and $\Phi_{\mathrm{TM}}(f, h)$ for different values of $f$ and $h$ centered around the initial guess values. For the computations we used the rigorous coupled-wave method ${ }^{18}$ for



(a)

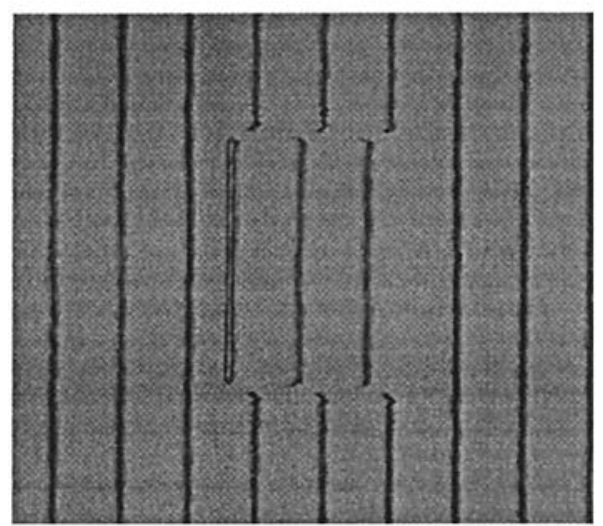

(b)

Fig. 2. Interferograms obtained for one of the gratings (a) TE polarization (b) TM polarization. Interferograms are approximately $500 \mu \mathrm{m} \times 500 \mu \mathrm{m}$ square. The phase shift observed between the two interferograms is a direct observation of the form birefringence of the subwavelength grating. For ease of interferogram exploitation note that nonlinear postprocessing was applied to the CCD pictures, which otherwise show regular sinusoidal patterns.

TE polarization and its enhanced version ${ }^{19}$ for $\mathrm{TM}$ polarization. Finally, the two equations

$$
\begin{gathered}
\Phi_{\mathrm{TE}}(f, h)-\Phi_{\mathrm{TE}}^{\text {meas }}=0, \\
\Phi_{\mathrm{TM}}(f, h)-\Phi_{\mathrm{TM}}^{\mathrm{meas}}=0,
\end{gathered}
$$

are solved. In Eqs. (3a) and (3b) $\Phi_{\mathrm{TE}}^{\text {meas }}$ and $\Phi_{\mathrm{TM}}^{\text {meas }}$, respectively, are the measured phase-shift data. Figure 3 shows the two curves in the configuration space corresponding to the $(f, h)$ values that satisfy Eqs. (3a) and (3b). It is obtained for the grating fabricated with a dose deviation of $60 \%$. The solid and the dashed curves correspond to TM and TE polarizations, respectively. The final solution for $f$ and $h$ is found at the intersection of the two curves. When estimating the parameters of the five gratings, we did not assume that these gratings were fabricated on the same substrate and considered five independent problems. The five fill factor (or equivalently linewidth) and depth values we obtained are given in the third and the fourth columns of Table 


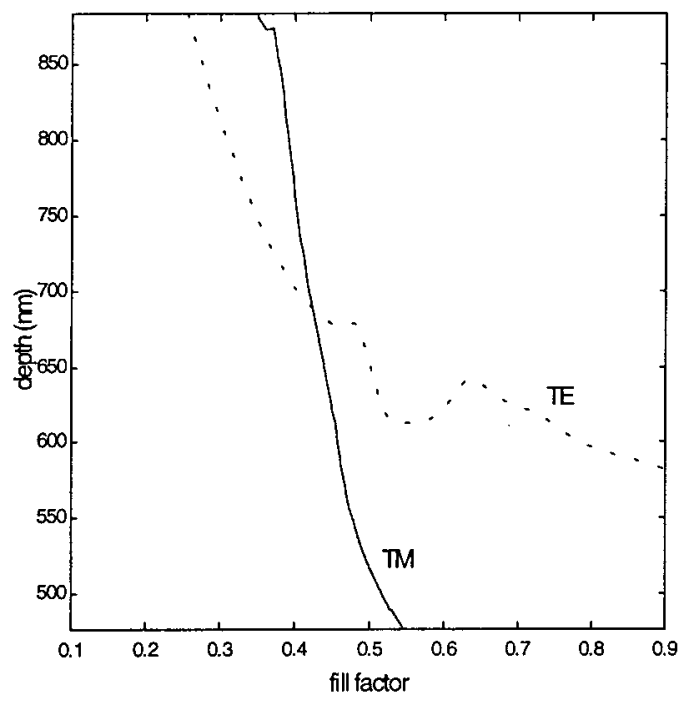

Fig. 3. Graphical resolution for the inverse problem and for the grating fabricated with a dose deviation of $60 \%$. Note the dashedcurve bend near $f=0.55$. This nonmonotonous behavior may be responsible for nonunique solutions. We believe that this is because the grating period considered here is larger than the cutoff and the structural cutoff values. Dashed curve, $\Phi_{\mathrm{TE}}(f, h)-\Phi_{\mathrm{TE}}^{\text {meas }}$ $=0$. Solid curve, $\Phi_{\mathrm{TM}}(f, h)-\Phi_{\mathrm{TM}}^{\text {meas }}=0$.

1. We note that, as expected, large linewidths are obtained for large doses.

After this study the substrate was covered with gold and examined with a SEM. From the SEM pictures the five grating linewidths were measured. The latter are reported in the second column of Table 1. We estimated that the linewidths are measured with an absolute accuracy of $\pm 5 \mathrm{~nm}$ typically or \pm 10 $\mathrm{nm}$ according to the quality of the SEM picture and according to the amount of scummy gold deposited. ${ }^{20}$ Basically, the SEM observation data are consistent with those predicted by inverse scatterometry. One of the SEM pictures shown in Fig. 4 was specifically dedicated to the estimation of the grating depth. For that purpose the grating was scratched with a pencil before the SEM observation. As a result, the grating ridges fell down, lying horizontally on the substrate. In this way perspective errors due to tilted SEM observations were suppressed. We found a depth of $693 \pm 8 \mathrm{~nm}$. Good agreement is again obtained. Figure 4 also confirms that the

Table 1. Experimental Data ${ }^{a}$

\begin{tabular}{rccc}
\hline $\begin{array}{c}\text { Dose } \\
(\%)\end{array}$ & $\begin{array}{c}\text { Linewidth }(\mathrm{nm}) \\
\text { SEM Data }\end{array}$ & $\begin{array}{c}\text { Linewidth } \\
(\mathrm{nm})\end{array}$ & $\begin{array}{c}\text { Grating Depth } \\
(\mathrm{nm})\end{array}$ \\
\hline-15 & 134 & 131 & 693 \\
0 & 143 & 150 & 685 \\
20 & 162 & 158 & 689 \\
40 & 178 & 170 & 688 \\
60 & 185 & 190 & 687 \\
\hline
\end{tabular}

${ }^{a}$ The second column provides measurements obtained with a SEM. The third and the final columns correspond to values obtained by numerical solution of the inverse problem.

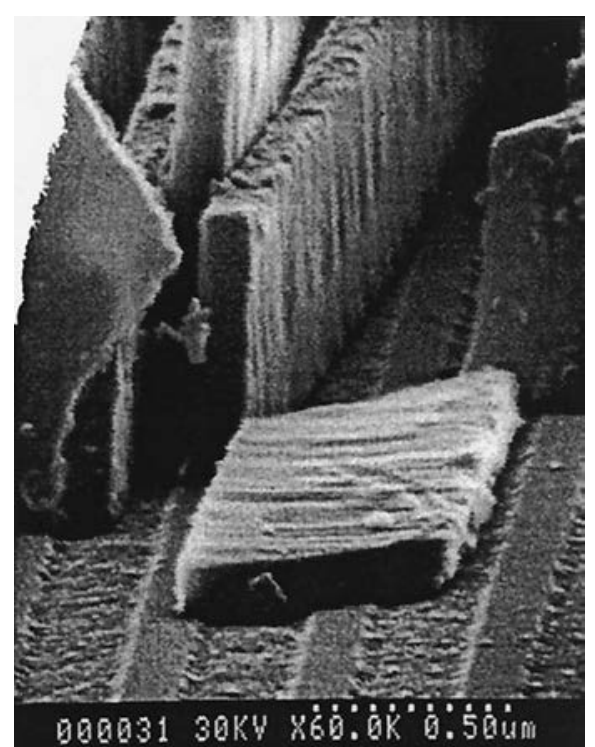

Fig. 4. SEM picture for estimating the grating depth and for validating the lamellar grating assumption.

etched depth and the $\mathrm{TiO}_{2}$ layer thickness are nearly identical. It is found that the gratings were slightly overetched with the reactive ion etching machine, the overetching depth being equal to a few nanometers. This observation in fine validates the lamellar grating model used in this experimental study.

\section{Discussion and Conclusion}

We proposed an interferometric method to estimate the linewidth and the etched depth of lamellar subwavelength gratings. The method is simple and relies on two interferometric measurements of the zero-transmitted order under two orthogonal polarizations. Its principle relies on the analogy between subwavelength lamellar gratings and homogeneous thin films. Since this analogy holds only for periods much smaller than the wavelength of the probing light, the method is not applicable for large periods. But, as shown in Section 2, there is no restriction concerning arbitrarily small periods. Concerning the computational time, the method is extremely fast, since only two quantities have to be computed.

The proposed method was tested with five lamellar gratings etched in a $\mathrm{TiO}_{2}$ layer deposited on a glass substrate with different linewidths. All gratings were fabricated on the same substrate and etched at the same time. Electromagnetic theory was used to compute the grating linewidths and etched depths, assuming five independent problems. Concerning the etched depth an average value of $688 \mathrm{~nm}$ in good agreement with SEM observations $(693 \pm 8 \mathrm{~nm})$ was found. Moreover, consistent with the fabrication process, rather similar values for the depths were obtained for the five gratings: The standard deviation was only $3 \mathrm{~nm}$, and the maximum deviation between the five measurements was $8 \mathrm{~nm}$. The measured linewidths were compared with those obtained from SEM observations. The maximum de- 
viation between the interferometric and the SEM measurements is $8 \mathrm{~nm}$, and the standard deviation is $6.3 \mathrm{~nm}$. It is interesting to relate these sensitivity values to fabrication errors of the polarizing beamsplitter grating that motivated this research. For this specific application electromagnetic computations indicate ${ }^{16}$ that a precise control of the linewidth $( \pm 4 \mathrm{~nm})$ and the etched depth $( \pm 10 \mathrm{~nm})$ are required for maintaining high extinction-ratio values larger than 100.

In this experimental study the inverse interferometric method was used for control after fabrication. It can also be applied in situ during the reactive ion etching process for monitoring the fabrication. Generally speaking, we believe that the insight gained from the static limit analogy may be useful for more complex grating geometries, such as lamellar gratings etched in multilayer Bragg reflectors, for example. Moreover, we also believe that the knowledge of the phase of the diffracted orders is important for inverse scatterometry, as was recently illustrated in the related context of super resolution in the microwave region of the spectrum. ${ }^{21}$

The authors are grateful to Jean Landreau and Alain Carenco from the Centre National des Etudes de Télécommunications for coating the $\mathrm{TiO}_{2}$ films. They thank Jean-Jacques Greffet from the Ecole Centrale at Chatenay-Malabry for fruitful discussions, Hugues Giovannini from the Laboratoire d'Optique des Surfaces et des Couches Minces at Marseille, and two anonymous reviewers for their helpful suggestions and comments. The authors also acknowledge Denis Joyeux, Raymond Mercier, Michel Mullot, and Bruno Bianchi from the Institut d'Optique for fruitful discussions, experimental help at different stages of this study, including interferometer design, Talystep profile measurements, and other interferometric techniques.

\section{References and Notes}

1. A. N. Tikhonov and V. Y. Arsenin, Solutions of Ill-Posed Problems (Winston, Washington, D.C., 1977).

2. A. Roger and M. Breidne, "Grating profile reconstruction by an inverse scattering method," Opt. Commun. 35, 299-302 (1980).

3. S. S. H. Naqvi, R. H. Krukar, J. R. McNeil, J. E. Franke, T. M. Niemczyk, D. M. Haaland, R. A. Gottscho, and A. Kornblit, "Etch depth estimation of large-period silicon gratings with multivariate calibration of rigorously simulated diffraction gratings," J. Opt. Soc. Am. A 11, 2485-2493 (1994).

4. N. Chateau, J. C. Saget, and P. Chavel, "Diffraction analysis and experimental investigation of reflection-free holographic phase gratings," Pure Appl. Opt. 2, 299-314 (1993).

5. Y. Cui and R. M. A. Azzam, "Applications of the normalincidence rotating-sample ellipsometer to high- and lowspatial-frequency gratings," Appl. Opt. 35, 2235-2238 (1996).

6. H. Giovannini, C. Deumié, H. Akhouayri, and C. Amra, "Angle- resolved polarimetric phase measurement for the characterization of gratings," Opt. Lett. 21, 1619-1621 (1996).

7. S. S. H. Naqvi, J. R. McNeil, R. H. Krukar, and K. P. Bishop, "Scatterometry and the simulation of diffraction based metrology," Microlith. World 2, 5-16 (1993).

8. K. P. Giapis, R. A. Gottscho, L. A. Clark, J. B. Kruskal, D. Lambert, A. Kornblit, and D. Sinatore, "Use of light scattering in characterizing reactively ion etched profiles," J. Vac. Sci. Technol. A 9, 664-668 (1991).

9. N. Blayo, R. A. Cirelli, F. P. Klemens, and J. T. C. Lee, "Ultraviolet-visible ellipsometry for process control during the etching of submicrometer features," J. Opt. Soc. Am. A 12, 591-599 (1995).

10. M. K. Minhas, S. A. Coulombe, S. S. H. Naqvi, and J. R. McNeil, "Ellipsometric scatterometry for the metrology of sub0.1- $\mu$ m-linewidth structures," Appl. Opt. 37, 5112-5115 (1998).

11. G. Bouchitté and R. Petit, "Homogenization techniques as applied in the electromagnetic theory of gratings," Electromagnetics 5, 17-36 (1985).

12. Strictly speaking, the Airy formula for thin films has to be used.

13. Ph. Lalanne, S. Astilean, P. Chavel, E. Cambril, and H. Launois, "Blazed-binary subwavelength gratings with efficiencies larger than those of conventional échelette gratings," Opt. Lett. 23, 1081-1083 (1998).

14. For the sake of illustration, see Fig. 1 in Ref. 13, where it is shown that, for periods larger than the structural cutoff, the phase of the zero order is not a monotonous function of the fill factor and exhibits a rather chaotic behavior.

15. $\mathrm{Ph}$. Lalanne and $\mathrm{D}$. Lemercier-Lalanne, "Depth dependence of the effective properties of subwavelength gratings," J. Opt. Soc. Am. A 14, 450-458 (1997).

16. Ph. Lalanne, J. Hazart, P. Chavel, E. Cambril, and H. Launois, "A transmission polarizing beam splitter grating," J. Opt. A: Pure Appl. Opt. 1, 215-219 (1999).

17. Strictly speaking, the phase shifts obtained from the interferograms are known modulo $2 \pi$. The indetermination is removed in practice by consideration of the small finite set of possible $2 \pi$ phase jumps and retention of only physical solutions. If one knows approximately the etched depth (as is the case for most characterization problems) or if in situ monitoring is considered, there is no ambiguity. In the latter case the absolute phase shift is observed in real time.

18. M. G. Moharam, E. B. Grann, D. A. Pommet, and T. K. Gaylord, "Formulation for stable and efficient implementation of the rigorous coupled-wave analysis of binary gratings," J. Opt. Soc. Am. A 12, 1068-1076 (1995).

19. Ph. Lalanne and G. M. Morris, "Highly improved convergence of the coupled-wave method for TM polarization," J. Opt. Soc. Am. A 13, 779-784 (1996).

20. For the SEM linewidth measurements the Au film deposited on the sample is a source of error. The additional thickness is difficult to estimate. However, because the gold coating of the vertical walls appears brigther and scummy in the SEM photographs, the additional thickness can be partially removed during estimation of the linewidth.

21. F. C. Chen and W. C. Chew, "Experimental verification of super resolution in nonlinear inverse scattering," Appl. Phys. Lett. 72, 3080-3082 (1998). 